\title{
La compétence argumentative dans les examens de certification officielle de FLE et ELE
}

\author{
The Argumentative Competence in the Official Certification \\ Examinations DELF and DELE
}

Géraldine Durand

Université de Salamanque, Espagne

Résumé : En fonction de la langue évaluée dans les examens de certification officielle pour l'obtention du DELF et du DELE B1 et B2, les exigences concernant le développement de la compétence argumentative chez le candidat vont être spécifiques. Conditionné par les normes auxquelles se plient les épreuves qui visent à la certification officielle d'un niveau, le contenu de la tâche attendu dans les exercices des sujets révèle une conception propre de la dimension argumentative que doit avoir un discours en français ou en espagnol.

Mots-clés : argumention, CECRL, DELF, DELE, niveaux B1-B2.

Abstract: Depending on the language assessed in the official certification examinations for the DELE and DELF B1 and B2, the requirements for the development of the candidate's argumentative competence will be specific. Determined by the rules guiding the examinations to obtain the official certification in a level, the task contents in exercises about topics reveal a specific conception of the argumentative dimension that a French or a Spanish discourse must have.

Keywords: argumention, CECRL, DELF, DELE, levels B1-B2.

\section{La conception de la communication dans le CECRL}

Le désir de rapprocher les peuples et de créer une citoyenneté européenne motive l'harmonisation de l'enseignement et de l'évaluation des apprentissages en langue étrangère proposée par le CECRL. Apprendre une langue étrangère participe au projet d'instaurer une communication fluide et rationnelle basée non seulement sur les points communs, mais aussi sur la compréhension et l'acceptation des différences.

Rapprocher l'enseignement du français et de l'espagnol, c'est rapprocher les Français et les Espagnols en transmettant deux langues romanes et deux cultures. Enseigner le FLE ou l'ELE, C'est développer chez l'apprenant la capacité d'adaptation à une langue et à une culture et développer ainsi une sensibilité particulière 
conditionnée par l'altérité. La priorité pour le CECRL est de former des esprits qui acceptent les différences et qui sont disposés à agir en médiateurs interculturels pour mieux se comprendre, tout en affirmant leurs opinions et leurs convictions et en évaluant de façon critique celles des autres, mais en restant neutres et pacifiques. Le CECRL invite à créer un contexte de médiation où « on est moins concerné par nos besoins, nos idées ou notre façon de nous exprimer que par ceux de la partie ou des parties pour laquelle ou lesquelles on médie " (CERCL, 2018, p. 109), ce qui suppose une compétence sociale évidente. Par ailleurs, ayant opté pour une approche actionnelle, le CECRL accepte une vision globale du discours qui est dialogale et où "la co-construction du sens' (grâce à l'interaction) [est] au centre du processus d'apprentissage et d'enseignement ॥ (CERCL, 2018, p. 28).

Le CECRL réunit, par conséquent, tous les ingrédients nécessaires pour avoir une conception de la communication où la "dimension argumentative occupe une place de choix [...] (particulièrement à partir du niveau B1) " (Doury \& Claudel, 2018, p. 35) parce que, dans ses descripteurs, comme dans les théories de l'argumentation, on part du principe qu'argumenter est, dans sa structure, une activité qui implique nécessairement au moins deux choses et deux personnes. Effectivement, d'une part, l'argumentation se caractérise par sa condition dialogique, c'est-à-dire qu'au minimum deux personnes se rencontrent : celle qui produit l'argument et celle qui le reçoit. Et d'autre part, deux choses sont mises en rapport : quelque chose est une raison pour une autre chose. Les raisons fournies constituent l'argument pour que le locuteur arrive à une conclusion et pour que le récepteur adopte cette conclusion. Pour le CECRL, le discours est donc par définition argumentatif.

Ainsi, la compétence argumentative est présente dans la plupart des échelles de descripteurs. Ressortent notamment celles portant sur toutes les activités langagières et surtout sur les activités de production, d'interaction et de médiation. Cependant, d'autres échelles sont impliquées: l'apprenant est amené à argumenter lorsqu'il développe sa compétence pluriculturelle et ses compétences communicatives langagières, en particulier ses compétences pragmatiques et aussi la sociolinguistique. Toutes ces échelles de descripteurs reprennent des composantes de la communication qui ne sont pas séparées et qui s'imbriquent l'une dans l'autre. C'est pourquoi, les notions propres à la compétence argumentative s'entrecroisent dans les échelles de descripteurs. Le CECRL intègre toutes les manifestations prototypiques de l'argumentation présentes dans les théories et il les adapte à une approche sociale de la langue et de la communication, situant ainsi l'argumentation dans le discours ef se rapprochant de la perspective adoptée par la logique naturelle, qui a une vision généralisée de l'argumentation dans le discours (Grize, 1990).

Cette approche se retrouve dans les examens officiels de FLE et ELE, qui permettent de certifier qu'est atteint chacun des niveaux marqués par le CECRL et ses échelles de descripteurs. Le processus d'enseignement, d'apprentissage et d'évaluation envisagé par le CECRL, sur lequel s'appuient les formats d'examen du DELF et du DELE, a pour objectif le développement des compétences générales et des compétences communicatives langagières. Ainsi, ce modèle se base sur une évaluation par compétences (Marcos García, 2009, p. 360) invitant l'apprenant à développer ses compétences en réalisant des activités langagières. Les échelles sont celles qui

\footnotetext{
${ }^{1}$ C'est le volume complémentaire du CECRL qui souligne en caractère gras.
} 
organisent l'apprentissage et fractionnent les activités et les compétences communicatives langagières.

L'observation des descripteurs du CECRL révèle que la capacité à argumenter n'est requise que très timidement au niveau $\mathrm{A} 2$ et de façon plus représentative à partir du niveau B2, car la compétence argumentative est cognitivement et linguistiquement exigeante. En effet, parmi les commentaires précédents toutes les échelles de descripteurs qu'offre le volume complémentaire du CECRL, dans celui portant sur le "monologue suivi : argumenter ", il est ouvertement reconnu que le niveau B2 est le " niveau auquel cette capacité [la capacité à défendre un argument] est un concept marquant $॥$ (2018, p. 75). Exposer des arguments et formuler un raisonnement complexe impliquent des compétences linguistiques développées permettant de rendre compte des nuances, des détails fins, des ambigüités, mais impliquent aussi des compétences générales, pluriculturelles, pragmatiques et sociolinguistiques. Le CECRL considère que l'utilisateur élémentaire des niveaux Al et A2 n'a pas à démontrer une grande capacité à argumenter. Sa connaissance de la langue étant limitée et sa formation inachevée, il ne peut pas faire appel aux ressources nécessaires qui peuvent être stratégiquement mises au service d'un discours persuasif élaboré. En revanche, chez les utilisateurs indépendants, surtout à partir du niveau B2 et évidemment chez les utilisateurs expérimentés, la compétence argumentative est très présente. À partir du niveau B2, les échelles de descripteurs du CECRL signalent que la maîtrise de cette compétence est déterminante pour démontrer que le niveau est atteint. Mais, déjà au niveau B1, dans certaines situations de communication, cet utilisateur doit être compétent pour « développer une argumentation suffisamment bien ॥ (2018, p. 75), même si la brièveté est acceptée. En effet, au niveau B1, deux échelles décrivent les compétences centrées sur la capacité à argumenter que doit développer l'utilisateur quand il doit produire des "Essais et rapports» ou un «Monologue suivi ». C'est pourquoi, notre attention va porter sur le niveau exigé des utilisateurs indépendants, c'est-à-dire sur les niveaux B1 et B2.

Concernant la certification officielle, les descripteurs sont, nous venons de le rappeler, la référence pour l'élaboration et l'évaluation des exercices. D'ailleurs, avec le Manuel visant à Relier les examens de langues au Cadre européen commun de référence pour les langues: Apprendre, enseigner, évaluer (CECRL), le Conseil de l'Europe cherche à mettre "en œuvre [les démarches pour] une compréhension commune des Niveaux communs de référence $"(2009$, p. 11). Cependant, selon la langue évaluée, la manière dont doit se concrétiser la compétence argumentative adopte une forme propre et donne lieu ainsi à des formats d'examens, où les exercices proposés, comme aux niveaux B1 et B2, montrent des nuances et des différences dans l'utilisation des descripteurs du CECRL et dans les intentions de l'enseignement des langues secondes.

\section{La compétence argumentative dans les examens de certification officielle}

Pour pouvoir procéder à l'analyse de la compétence argumentative dans les examens de certification officielle, nous n'examinerons pas les épreuves proposées pour évaluer la compréhension parce que la compétence argumentative est plus présente dans les descripteurs du CECRL de la production. En effet, comme la forme du discours détermine son efficacité argumentative, les compétences pragmatiques sont inévitablement sollicitées dans une situation de communication qui envisage 
l'expression d'au moins un point de vue. Le CECRL développe six échelles de descripteurs pour les compétences pragmatiques qui vont être plus centrées sur l'expression que sur la compréhension. Elles concernent des aspects de capacités particulières de l'apprenant pour intervenir dans une situation de communication qui peut être résolument argumentative, à savoir la souplesse, les tours de parole, le développement thématique, la cohérence et la cohésion, la précision et l'aisance à l'oral.

Seules les épreuves qui demandent une production qu'elle soit écrite ou orale seront donc pertinentes pour notre étude. Par ailleurs, pour les niveaux B1 et B2 ${ }^{2}$ évalués dans les examens du DELF et du DELE, les modèles d'examens seront notre référence. Pour les examens du DELF, les grilles d'évaluation et les diapositives des présentations de la plateforme d'autoformation des examinateurs et des correcteurs du DELF et du DALF apporteront des informations complémentaires. De plus, les exercices et les données à traiter se multipliant, nous proposons deux tableaux que nous avons élaborés pour chacun des niveaux B1 et B2, pour que cette étude soit plus claire et visuelle.

\subsection{Le niveau $B 1$}

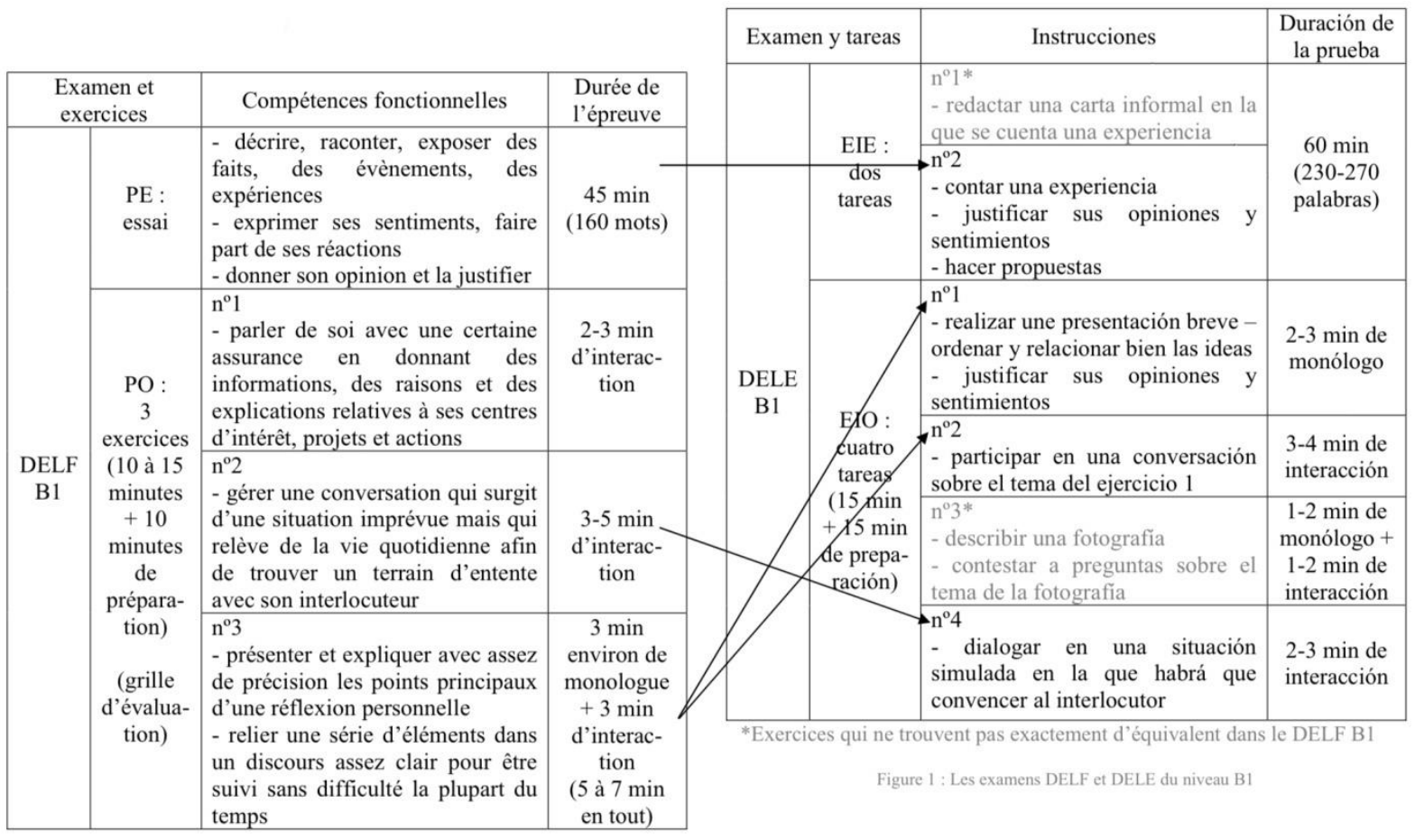

\section{La compétence argumentative dans la production écrite des examens du DELF et du DELE du niveau $B 1$}

Lors de l'épreuve de production écrite pour le DELF B1, le candidat dispose de quarante-cinq minutes pour réaliser un seul exercice qui consiste en la rédaction d'un essai de 160 mots minimum. Dans un texte construit et cohérent, ce pour quoi il peut obtenir jusqu'à trois points sur vingt-cinq, il doit pouvoir montrer qu'il est capable de

\footnotetext{
2 Voir annexes.
} 
présenter des faits en décrivant, en racontant, en exposant des faits, des évènements ou des expériences. Ceci correspond à la capacité à produire des discours factuels basés sur la narration et la description. Mais, il doit aussi pouvoir montrer qu'il est capable d'exprimer sa pensée comme étant le fruit d'une réflexion puisqu'il est amené à exprimer ses sentiments, à faire part de ses réactions, à donner son opinion et à la justifier. Ceci correspond à la capacité à produire un discours argumentatif. Ces deux critères peuvent recevoir chacun un maximum de quatre points sur vingt-cing tandis qu'au niveau A2, la capacité à donner ses impressions ne reçoit que la moitié de ces points puisque la capacité à raconter et à décrire est, comme dans les échelles de descripteurs du CECRL, celle qui est priorisée. Le niveau $\mathrm{B} 1$ se trouve réellement à mi-chemin entre le niveau A2 et le niveau B2 : la capacité à raconter a autant de poids que la capacité à argumenter et les faits sollicités peuvent avoir la fonction d'arguments dans le récit d'expériences dont la valeur est justificatoire de l'opinion. Le récit d'expériences personnelles revêt la valeur d'argument. Comme dans la perspective de la logique naturelle, « le fait est le meilleur des arguments $॥$ (Grize, 1990, p. 44). La description et la narration interviennent dans la construction du point de vue et endossent alors une valeur argumentative.

Pour faire face à l'épreuve d'expression et d'interaction écrites, le candidat au DELE Bl dispose de soixante minutes, c'est-à-dire quinze minutes de plus que le candidat au DELF B 1, mais il doit réaliser deux tâches et écrire un total de 230-270 mots. Globalement, le texte à produire est plus long, mais il s'agit d'une production semi-guidée. Le premier exercice requiert la compétence discursive de la description et surtout de la narration, tandis que la capacité à se justifier est sollicitée dans le deuxième exercice qui invite à raconter une expérience personnelle, mais dans une approche différente de celle de l'examen du DELF B1. L'examen du DELF B1 laisse une large place à l'expression du point de vue du candidat qui s'appuie sur le récit d'expériences personnelles. Le DELE BI sollicite aussi la capacité à raconter des faits vécus, mais l'objectif n'est pas celui de donner une opinion pour influencer le destinataire; il s'agit simplement de fournir des données informatives, limitant ainsi l'exigence concernant la compétence argumentative. Quantitativement, l'examen du DELE privilégie la capacité à raconter alors qu'à ce niveau, l'examen du DELF exige la maîtrise des principes basiques de la narration et de l'argumentation.

\section{La compétence argumentative dans la production orale des examens du DELF et du DELE du niveau B1}

Lors de l'épreuve de production orale du DELF Bl, le candidat est soumis à trois exercices qui se succèdent. Après la prise de contact qui constitue le premier exercice, le deuxième exercice est une conversation avec l'examinateur qui vise à gérer une situation imprévue, mais qui relève de la vie quotidienne et dont la finalité est de trouver des solutions à un problème et un terrain d'entente avec l'interlocuteur. Cet exercice est une tâche qui consiste à résoudre un problème qui peut surgir dans la vie réelle et il fait donc appel à la capacité persuasive du candidat qui s'établit sur la base de la médiation et de l'interaction et où interviennent le savoir-être et le savoirfaire du candidat afin que la conversation soit rationnelle et raisonnable, partageant ainsi le même objectif que l'approche pragma-dialectique de l'argumentation. Même si le nom de l'épreuve ne le dévoile pas, cet exercice intègre donc plusieurs activités langagières, tout comme le troisième exercice. Le troisième exercice fait aussi appel à la capacité à persuader du candidat, mais le format est différent. II ne s'agit 
plus de résoudre un problème de la vie quotidienne, mais de faire part d'une réflexion personnelle et d'exprimer un point de vue dans un monologue de trois minutes environ. Le monologue est suivi de trois autres minutes d'interaction entre le candidat et l'examinateur. II peut prendre la forme d'un débat, au cours duquel le candidat peut donner des détails sur ce dont il a parlé dans le monologue et confronter son opinion à d'autres visions de la même réalité. Tel que le signale le deuxième critère de la grille d'évaluation pour cet exercice, le candidat est amené à "présenter et à expliquer avec assez de précision les points principaux d'une réflexion personnelle ॥ qui naît à partir d'un bref texte déclencheur, intégrant ainsi à l'épreuve une activité de compréhension écrite et créant une situation de médiation de textes. Pour répondre au troisième critère de la grille, il doit aussi être capable de « relier une série d'éléments dans un discours assez clair pour être suivi sans difficulté la plupart du temps ॥. Cet exercice appelle à la maîtrise de la compétence discursive qui implique un discours structuré, cohérent et organisé comme celvi préconisé par les théories de l'argumentation quand elles se réfèrent à la dispositio. Mais, il appelle aussi à la capacité à défendre son point de vue et à confirmer ses idées, bien que ce ne soit que succinctement. Ce dernier exercice est un avant-goût de celui que le candidat va réaliser au niveau au-dessus : ici, de par le temps imparti, il se limite à la formulation d'une opinion qui peut se voir confrontée, lors de l'interaction, à de possibles objections ou simplement au besoin d'éclaircissements de l'interlocuteur pour lever toutes les ambigüités, s'il y a lieu. II n'est pas encore exigé du candidat de parvenir à produire une argumentation bien développée, mais l'examen français demande déjà au niveau $\mathrm{Bl}$ une certaine maîtrise de la compétence argumentative, alors que celle-ci est prédominante dans les échelles de descripteurs du CECRL à partir du niveau B2.

Comme l'épreuve de production orale du DELF B1, l'épreuve d'expression et d'interaction orales du DELE Bl est une épreuve individuelle où le candidat se présente face à l'examinateur. Pour les deux langues, ces épreuves durent quinze minutes et un nombre similaire d'exercices est proposé : en français trois et en espagnol quatre. Comme pour l'examen du DELF, les situations de communication proposées pour le DELE se succèdent, hachent et fractionnent le temps imparti. Le troisième exercice est celui qui ne trouve pas d'équivalent dans l'examen français, car il fait appel à la capacité à décrire du candidat, une capacité qui n'est plus exactement ciblée dans l'examen français si elle n'est pas accompagnée de la capacité à argumenter. En revanche, une claire correspondance peut être établie entre les autres exercices. Le quatrième exercice du DELE B 1 correspond au deuxième exercice du DELF Bl établissant une interaction dont le but est d'atteindre le consensus à l'issue de l'échange. Donc, la compétence de la médiation est requise et doit être accompagnée d'un haut degré de coopération et de collaboration, sans quoi la conversation ne peut aboutir. La différence se trouve dans le temps imparti, qui est inférieur pour l'examen du DELE, ce qui implique inévitablement que le processus pour trouver un terrain d'entente sera plus superficiel. Quant aux deux premiers exercices du DELE B1, ils impliquent la mobilisation des mêmes compétences de la part du candidat que le dernier exercice du DELF B1, car il dispose du même temps, entre cinq à sept minutes, pour produire d'abord, en deux ou trois minutes, un discours structuré avec une introduction, un développement et une conclusion, où les idées exposées sont présentées de façon organisée et cohérente, montrant un esprit rationnel, comme le décrivent les théories de l'argumentation. II doit ensuite interagir 
avec l'examinateur et établir une conversation pendant trois à quatre minutes. Dans cette phase d'interaction, la perspective est légèrement différente par rapport à l'exercice correspondant à l'examen du DELF Bl qui, de par son nom, doit être propice à l'uexpression d'un point de vue» donnant la possibilité de prendre position clairement. Le format des deux examens est extrêmement proche, mais dans cet exercice de l'examen du DELE B1, l'interaction ne repose pas sur l'éclaircissement d'idées ou sur la confrontation d'opinions née d'une réflexion plus ou moins profonde. Elle consiste plutôt à partager des opinions nées d'appréciations. Les questions que peut poser l'examinateur de l'examen du DELE invitent peu à la confrontation d'opinions et favorisent l'expression d'appréciations personnelles centrées sur les goûts ou les habitudes. En effet, les sujets proposés dans les examens de certification officielle se caractérisent par leur neutralité : les objections émises à une opinion ne peuvent être ni blessantes ni discriminatoires et aucun groupe, aucune communauté, aucune nation, quelles que soient ses valeurs, ses croyances, ses convictions, sa culture et ses références, ne doivent se sentir visés et jugés. Cependant, dans l'examen du DELE, ce souci de neutralité poussé à l'extrême conditionne la possibilité de développer pleinement la compétence argumentative. Les questions posées par l'examinateur de l'examen du DELE permettent d'éviter la confrontation d'idées opposées où règne le désaccord qui devrait être résolu et qui implique l'affichage de convictions personnelles, tandis que les questions auxquelles est soumis le candidat au DELF doivent lui donner la possibilité de « détailler des points abordés au cours de son exposé et discuter de son point de vue " (diapositive 6 de la présentation pour l'évaluation de la production orale).

Plus que les épreuves de production écrite, les épreuves de production orale des examens qui certifient le niveau $\mathrm{Bl}$ en français et en espagnol accordent une place non négligeable à la capacité à argumenter, bien que cette capacité ne soit pas représentative dans les échelles de descripteurs du CECRL à ce niveau-là. Finalement, la différence du traitement de la compétence argumentative ne réside pas dans une interprétation différente des descripteurs du CECRL, mais elle est essentiellement due aux questions de l'examinateur qui se prêtent plus ou moins au développement de cette compétence. Par conséquent, globalement, les exigences de l'examen du DELE B1 portent plus que l'examen du DELF B1 sur la capacité à décrire et à raconter, reflétant parfaitement les descripteurs du CECRL pour le niveau B 1, où la compétence argumentative commence à être présente, mais où la capacité à raconter et à décrire reste encore prédominante, et insistant plus, dans la communication, sur la transmission de faits que d'idées. Deux exercices que propose l'examen français, l'un à l'écrit et l'autre à l'oral (le troisième) appliquent les descripteurs du niveau Bl de deux échelles dont la situation de communication est spécialement marquée par l'intention argumentative, à savoir "Essais et rapports" et "Monologue suivi : argumenter ». Cette exigence montre un intérêt particulier dans l'examen du DELF B1 pour la compétence discursive, qui facilite la transmission de la réflexion faite rationnellement. Cet intérêt semble être une constante dans les examens français, qui cherchent à évaluer le niveau d'un candidat en langue seconde et qui semblent hériter la philosophie des pratiques mises en place par l'enseignement et l'évaluation du français langue première. En effet, les exercices proposés aux examens externes du cycle terminal du lycée en langue première en France, tels que le commentaire de texte ou la dissertation, ne se limitent pas à l'évaluation des compétences strictement linguistiques et montrent la grande place accordée à la compétence 
argumentative. Quand le français est langue première, l'enseignement et l'évaluation proposés par le système éducatif insistent sur la formation de l'esprit critique et, quand il est langue seconde, l'accent est mis sur la formation de l'esprit rationnel en intégrant les différentes dimensions du discours à l'argumentation, s'inscrivant ainsi dans la lignée de la logique naturelle. En revanche, dans l'évaluation de la maîtrise de la langue espagnole langue première ou langue seconde, l'accent est mis sur la formation des esprits qui doivent être avant tout raisonnables. Le respect des opinions des autres est priorisé à tel point que la défense des convictions personnelles s'en trouve moins approfondie.

\subsection{Le niveau $B 2$}

\begin{tabular}{|c|c|c|c|c|c|c|}
\hline \multicolumn{2}{|c|}{$\begin{array}{c}\text { Examen et } \\
\text { exercices }\end{array}$} & \multirow{3}{*}{$\begin{array}{l}\text { Compétences fonctionnelles } \\
\text { - faire preuve d'une assez bonne } \\
\text { maîtrise code } \\
\text { sociolinguistique } \\
\text { - présenter des faits, un contexte, } \\
\text { une situation } \\
\text { - formuler clairement des idées } \\
\text { et arguments } \\
\text { - proposer des exemples pour } \\
\text { illustrer idées et arguments } \\
\text { - produire un texte cohérent et } \\
\text { clairement articulé }\end{array}$} & \multirow{3}{*}{$\begin{array}{c}\text { Durée de } \\
\text { l'épreuve } \\
\\
1 \text { heure } \\
\text { (250 mots) }\end{array}$} & Examen y tareas & Instrucciones & $\begin{array}{c}\text { Duración de } \\
\text { la prueba }\end{array}$ \\
\hline \multirow{6}{*}{$\begin{array}{c}\text { DELF } \\
\text { B2 }\end{array}$} & \multirow{2}{*}{$\begin{array}{l}\text { PE : } \\
\text { écrit } \\
\text { argu- } \\
\text { menté }\end{array}$} & & & $\begin{array}{l}\text { EIE: } \\
\text { dos } \\
\text { tareas }\end{array}$ & $\begin{array}{l}\mathrm{n}^{\circ} 1 \\
\text { - redactar una carta formal en la } \\
\text { que se explica una situación y se } \\
\text { opina acerca de ella } \\
\mathrm{n}^{\circ} 2^{*} \\
\text { - comentar datos de un documento } \\
\text { y expresar su opinión }\end{array}$ & $\begin{array}{l}80 \min \\
(300-360 \\
\text { palabras })\end{array}$ \\
\hline & & & & \multirow{4}{*}{$\begin{array}{l}\text { EIO : } \\
\text { tres } \\
\text { tareas }\end{array}$} & \multirow{2}{*}{$\begin{array}{l}\mathrm{n}^{\circ} 1 \\
\text { - hablar de las ventajas e } \\
\text { inconvenientes de una serie de } \\
\text { soluciones propuestas para una } \\
\text { situación determinada } \\
\text { - conversar sobre el tema }\end{array}$} & \multirow[t]{2}{*}{$\begin{array}{l}\text { 3-4 min de } \\
\text { monólogo } \\
+3 \text { min de } \\
\text { interacción }\end{array}$} \\
\hline & \multirow{4}{*}{$\begin{array}{c}\text { PO : } \\
\text { un seul } \\
\text { exercice }\end{array}$} & \multirow{4}{*}{$\begin{array}{l}\text { - dégager le thème de réflexion } \\
\text { et introduire le débat } \\
\text { - présenter un point de vue en } \\
\text { mettant en évidence des } \\
\text { éléments significatifs et / ou des } \\
\text { exemples pertinents } \\
\text { - marquer clairement les } \\
\text { relations entre les idées } \\
\text { - confirmer et nuancer ses idées } \\
\text { et ses opinions des précisions } \\
\text { - apporter des } \\
\text { - réagir aux arguments et } \\
\text { déclarations d'autrui pour } \\
\text { défendre sa position }\end{array}$} & \multirow{4}{*}{$\begin{array}{l}15-20 \text { min : } \\
\text { monologue } \\
+ \text { interaction } \\
\text { (débat) }\end{array}$} & & & \\
\hline & & & & & $\begin{array}{l}\mathrm{n}^{\circ} 2 * \\
-\quad \text { imaginar una situación y } \\
\text { describirla } \\
\text { - conversar acerca de sus } \\
\text { experiencias y opiniones sobre el } \\
\text { tema de la situación }\end{array}$ & $\begin{array}{l}2-3 \text { min de } \\
\text { monólogo } \\
+3 \text { min de } \\
\text { interacción }\end{array}$ \\
\hline & & & & & $\begin{array}{l}\mathrm{n}^{\mathrm{o}} 3^{*} \\
\text { - conversar sobre los datos de una } \\
\text { encuesta, expresando su opinión al } \\
\text { respecto }\end{array}$ & $\begin{array}{l}3-4 \text { min de } \\
\text { interacción }\end{array}$ \\
\hline & & & & qui ns & uvent pas exactement d'équivalent dans la & DELF B2 \\
\hline
\end{tabular}

\section{La compétence argumentative dans la production écrite des examens du DELF et du DELE du niveau B2}

Un candidat au DELF qui certifie qu'il a atteint le niveau B2 est capable, aussi bien à l'oral qu'à l'écrit, d'utiliser les stratégies argumentatives. Ce qui est une mise en bouche au niveau B1 est une réalité au niveau B2. À l'écrit, il dispose d'une heure pour produire un texte de 250 mots et il doit «faire preuve d'une assez bonne maîtrise du code sociolinguistique ") (diapositive 3). Comme au niveau B 1, l'influence de la logique naturelle se fait ressentir parce que la capacité à présenter des faits est intégrée dans la compétence argumentative, concédant aux faits une valeur justificatoire. Dans cet examen aussi, cette capacité a le même poids que la capacité à argumenter une prise de position, qui était limitée à l'expression de la pensée au niveau B1. Désormais, le candidat doit pouvoir " présenter des faits, un contexte, une situation, formuler clairement des idées et des arguments" (diapositive 3) "en soulignant de manière appropriée points importants et détails pertinents" (grille) et en proposant des exemples pour illustrer son argumentation. Ses arguments ne peuvent plus se limiter à un récit d'expériences. Cependant, le critère qui prédomine est celui de la cohérence 
et de la cohésion, qui a quatre points pour lui tout seul. L'accent est vraiment mis sur la rigueur à adopter lors de la présentation d'une position qui se veut avant tout rationnelle.

Comme pour l'examen du DELF B2, le candidat au DELE B2 se soumet à une épreuve qui demande de produire un texte écrit où un point de vue est développé dans un cadre formel. Concernant le format, à l'écrit de l'épreuve du B2, la situation est comparable à celle de l'épreuve du B1. Le candidat au DELE B2 doit faire face à un exercice de plus que le candidat au DELF du même niveau. Cet exercice supplémentaire est l'exercice 2. Il dispose de dix minutes de plus, mais il doit produire entre 50 et 110 mots de plus en s'appuyant sur des consignes qui marquent clairement toutes les étapes de la rédaction. La capacité argumentative du candidat est également sollicitée, mais elle doit être pour l'examen du DELE certainement moins développée que pour celui du DELF puisque la production écrite du DELF n'est pas semi-guidée et elle est plus longue que son analogue qui se trouve dans l'exercice 1. Les compétences pragmatiques n'ont pas autant de poids que dans l'examen français où ces compétences représentent plus de la moitié de la note de la production écrite. Dans l'examen espagnol, les compétences linguistiques et la médiation de textes prennent le pas sur les compétences pragmatiques. En effet, l'examen du DELE insiste davantage sur le fait que la communication intègre plusieurs activités langagières. Le modèle 0 d'examen pour la production écrite montre comment la production d'un texte écrit requiert la compétence de l'interaction et de la médiation, car le candidat est amené à comprendre un enregistrement, à prendre des notes, à traiter l'information pour sa production, mais aussi à analyser et à interpréter les données d'un graphique ou d'un programme d'une salle de théâtre.

\section{La compétence argumentative dans la production orale des examens du DELF et du DELE du niveau B2}

Quant à l'épreuve orale de l'examen du DELF, elle se déroule en deux temps. D'abord, dans un monologue suivi, le candidat dispose de cing à sept minutes pour défendre un point de vue argumenté sur un sujet proposé dans un texte déclencheur. C'est alors qu'il doit montrer qu'il peut dégager le thème de réflexion à partir d'un texte et introduire le débat, mais surtout qu'il peut " présenter un point de vue en mettant en évidence des éléments significatifs et/ou des exemples pertinents " (grille) en marquant clairement les relations entre les idées. L'exigence porte donc sur la capacité à développer et à organiser un discours. Le candidat est amené à prendre un soin particulier à choisir les idées qu'il veut développer pour qu'elles soient pertinentes et pour que le rapport qui les unit permette de produire un raisonnement cohérent. En plus de devoir faire preuve d'esprit critique, le candidat doit surtout respecter la maxime III du principe de coopération, qui s'inscrit dans la théorie de la conversation de Paul Grice (Escandell Vidal, 1996, pp. 93-94). Cette maxime met plus l'accent sur le contenu de ce qui est avancé que la manière dont ce contenu est formulé. Rappelons que Grice insiste sur l'importance du rapport qu'il doit y avoir entre ce qu'on dit et le sujet dont on discute. Ensuite, lors du débat avec l'examinateur, qui peut durer dix minutes environ, le candidat est invité à « confirmer et [à] nuancer ses idées et ses opinions ॥ (grille), à répondre à des questions qui sont posées pour qu'il montre s'il peut apporter des précisions et « réagir aux arguments et aux déclarations d'autrui pour défendre sa position ॥ (grille). Déjà à ce niveau-là, la capacité à apporter des précisions et des nuances est requise, marquant la rencontre avec 
l'examinateur du sceau de la conciliation, étant donné que les opinions catégoriques et fermées n'ont pas leur place. Cette épreuve orale met donc le candidat dans une situation fortement argumentative, qui est en accord avec la grande majorité des descripteurs du niveau B2 du CECRL. Les intervenants se trouvent dans une confrontation dialogique au sein d'un discours qui se co-construit et qui est facilement binaire, mais qui incite à prendre en compte l'autre. La capacité à écouter et à comprendre l'autre, à envisager d'autres points de vue est placée au centre de cette épreuve. Comme au niveau B1, ce format d'exercice ne priorise pas la formation de l'esprit critique, mais plutôt l'esprit de coopération pour la construction du discours, restant fidèle à la philosophie du CECRL et de ses objectifs généraux.

À l'oral, la durée de l'épreuve est la même pour les candidats au DELF B2 que pour ceux au DELE B2. Pourtant, le nombre d'exercices est bien différent, ce qui a une conséquence directe sur les attentes à avoir. Le candidat au DELE B2 est soumis à trois exercices. Comme lors de la production écrite, dans l'examen du DELE B2, I'accent est mis sur la capacité à interpréter des documents et à utiliser les données fournies. Dans le deuxième exercice, la compétence argumentative est secondaire et dans le troisième, elle est davantage présente, mais elle n'est pas prioritaire. En effet, dans ce dernier exercice, l'accent est plutôt mis sur l'art de la conversation : il s'agit d'échanger des appréciations, des interprétations et même des convictions. Le format du DELE suppose une rigueur argumentative moindre par rapport au DELF. Le point de départ n'est pas, comme à l'épreuve du DELF, une réflexion élaborée qui nourrit ensuite l'échange avec l'examinateur. Le temps imparti au candidat au DELE B2 pour les moments d'interaction ne permet pas comme pour l'examen du DELF de partager des opinions qui sont amenées à être nuancées. L'examen du DELE demande à développer la compétence argumentative de façon plus succincte et plus brève. Le format du premier exercice correspond exactement à celui proposé pour le DELF, mais le temps imparti est divisé de plus de moitié.

Malgré la référence des échelles de descripteurs du CECRL qui se retrouve dans les examens du DELF et du DELE, des différences qui surviennent entre les deux montrent que l'examen du DELF accorde une place prépondérante à la compétence argumentative, qui se développe à partir d'une réflexion élaborée sur des sujets où différentes opinions peuvent facilement être confrontées et qui invitent à prendre position tout en nuançant les propos. L'examen du DELE B2 se montre plus exigeant dans l'interprétation et le traitement de données que dans le développement de la compétence argumentative, le temps imparti ne permettant pas d'approfondir la réflexion. Comme en espagnol langue première, la communication en espagnol langue seconde se nourrit plus de données et de connaissances du sujet que d'appréciations basées sur la réflexion. L'enseignement des langues secondes, s'il veut répondre à la préparation des examens qui visent à certifier officiellement un niveau, peut s'inscrire dans la lignée de l'enseignement de la langue première favorisant, en fonction de la langue, la réflexion ou le traitement des données. Mais, dans les deux cas, la présence de la philosophie du CECRL est fortement perceptible, notamment dans l'assimilation des manifestations prototypiques de l'argumentation étudiées par les théories, mais aussi dans l'assimilation du concept de co-construction du discours proposé par la logique naturelle, qui envisage l'argumentation comme étant généralisée dans le discours. 


\section{Considérations finales}

L'analyse des échelles de descripteurs du volume complémentaire du CECRL rend évident que le CECRL perçoit la compétence argumentative comme centrale dans la communication. Pour le CECRL, le discours est argumentatif par définition. Comme en logique naturelle, la perspective adoptée est celle de voir l'argumentation généralisée dans le discours. Le CECRL montre toute la complexité de la communication humaine: ainsi, dans les épreuves des examens de certification officielle du DELF et du DELE, notamment aux niveaux B1 et B2, plusieurs activités langagières sont intégrées dans une même situation et s'entrecroisent de nombreuses compétences qui doivent être développées afin que les échanges aboutissent. C'est pourquoi, l'enseignement et l'apprentissage des langues secondes, s'inscrivant dans l'approche actionnelle, peuvent ne pas se limiter au développement des compétences linguistiques et peuvent contribuer à la formation intégrale de l'apprenant, qui est perçu comme une personne dont les agissements ont des répercutions dans la société. Dans la perspective du CECRL et des examens de certification officielle étudiés, l'apprenant est considéré comme un acteur social. D'ailleurs, cette approche actionnelle préconisée par le CECRL rappelle l'approche envisagée à l'Antiquité pour la formation des citoyens et la conception de la communication alors adoptée. C'est l'Antiquité qui a vu naître les théories de l'argumentation pour répondre aux besoins de la société qui demandait des citoyens impliqués et qui sachent faire entendre leur voix grâce à leurs discours persuasifs où était mis en œuvre l'art de penser et de bien parler. C'est à l'Antiquité que la rhétorique, alors qu'elle est mise au service de la formation des orateurs, est placée au centre de l'éducation. Suivant les pas d'Aristote, qui revêt la rhétorique d'une large dimension morale et éthique, le CECRL, lui, considère le développement des compétences générales, dont le savoir-être, comme un aspect qui marque le développement des compétences communicatives et le déroulement des activités langagières. II n'est donc pas étonnant d'observer que, selon les échelles de descripteurs, la compétence pluriculturelle intervient dans toutes les activités langagières et dans le développement de la compétence argumentative, dans un contexte pluriel où prédomine la diversité. La personnalité et d'autres facteurs personnels et comportementaux affectent la communication en général et le discours persuasif en particulier et exercent une influence sur la rationalité de la conversation. Comme la théorie de la conversation et la pragma-dialectique qui dictent des règles d'un échange communicatif aux participants (Eemeren \& Grootendorst, 2004, pp. 187-196), le CECRL pose aussi les principes qui régissent la conversation, mais en d'autres termes, car il s'appuie sur le caractère raisonnable et sur l'éthique de l'utilisateur comme acteur social. En effet, dans le CECRL, la langue est un phénomène social, elle fonctionne "dans sa dimension sociétale » (2018, p. 143). La langue est un outil que les citoyens utilisent pour agir dans la société et elle est aussi porteuse d'une culture. La langue-culture se sert de connaissances et d'habiletés spécifiques de la compétence sociolinguistique, car elle est empreinte des caractéristiques de la société dans laquelle elle est utilisée. Le CECRL voit une « nature sociale de l'apprentissage et de l'usage de la langue ॥ (2018, p. 27) et il privilégie la perspective plus dialectique de l'argumentation. Aussi dote-t-il l'enseignement des langues secondes d'une grande responsabilité dans la formation des citoyens. C'est alors qu'un fort lien s'établit entre les théories de l'argumentation et le CECRL : les deux préconisent des échanges compréhensibles, rationnels, cohérents et raisonnables afin 
d'arriver à une bonne entente entre les participants qui sont amenés à se prendre en compte mutuellement. II s'avère que la composition d'un discours persuasif et argumenté est une tâche où converge cette philosophie qui prône la tolérance etl'acceptation des différences. En effet, les visées de l'utilisation de l'argumentation et de l'utilisation des langues étrangères se rencontrent. En argumentant, les utilisateurs de la langue française et de la langue espagnole participent à la création d'un terrain d'entente pour arriver à un consensus où différents points de vue peuvent cohabiter.

Malgré ces intérêts communs et la présence des notions inhérentes à l'argumentation dans le CECRL, les examens de certification officielle des langues secondes, notamment l'examen du DELF et celui du DELE, n'insistent pas de la même manière sur la mobilisation de la compétence argumentative. Les variations ne sont pas dues aux possibles différences d'interprétation des descripteurs du CECRL, car les deux examens présentent des exercices qui ciblent les mêmes descripteurs. Elles ne sont dues ni au type d'exercices proposé, ni à des exigences différentes concernant le développement des compétences générales comme celle du savoir-être ou des compétences communicatives comme la compétence pluriculturelle ou sociolinguistique, car elles ne s'inscrivent pas dans le cadre didactique du processus d'enseignement, d'apprentissage et d'évaluation. Elles sont dues à des différences de format dans les sujets d'examen. Ce sont effectivement les questions que peut poser l'examinateur et le temps imparti qui conditionnent le degré de développement de la compétence argumentative. Comme dans l'évaluation de la langue première, les examens français proposent une vision globale de l'argumentation qui embrasse les perspectives logique, dialectique et rhétorique, car ils placent la construction d'une réflexion élaborée au centre de l'enseignement et de l'apprentissage et ils sollicitent la capacité à démontrer un raisonnement en ayant recours à une perception rationnelle des échanges. Le côté rationnel est celui qui régit la communication réussie. Dans les sujets des examens du DELE, les questions permises montrent une certaine pudeur lorsqu'il s'agit de l'expression de ses convictions et ceci affecte le degré de développement de la compétence argumentative. Comme en langue première, le candidat est invité à faire part d'une réflexion moins détaillée et moins engagée. Mais, cette réflexion est guidée par un esprit raisonnable qui se doit de respecter les opinions des autres en prenant toutes les précautions possibles. Puisque les exercices en interaction cherchent à éviter le conflit et les situations qui peuvent générer un désaccord ou des tensions, l'effort n'est pas centré sur l'élaboration d'une réflexion pour convaincre l'autre, mais plutôt sur la formulation brève d'une opinion qui peut prendre corps grâce au traitement de données. Le caractère raisonnable du participant est celui qui est encouragé. Les variations portant sur le traitement de la compétence argumentative dans les examens proposés pour la certification officielle des niveaux en FLE et ELE se situent donc dans le cadre des cultures éducatives présentes en France et en Espagne. La culture éducative propre à chaque pays laisse une empreinte sur les objectifs d'enseignement et ouvre la porte à l'existence d'exigences qui incitent la production de discours marqués par une variabilité culturelle en fonction du pays (Hidden, 2009a, p. 117, 2009b, p. 102). L'enseignement et l'évaluation des langues secondes portent donc le sceau de l'enseignement de la langue première. 


\section{Bibliographie}

Claudel, C., \& Doury, M. (2018). Les arbres argumentatifs comme supports à l'enseignement de l'argumentation en FLE. Mélanges CRAPEL, 39, pp. 33-53. https://www.atilf.fr/wp-content/uploads/publications/MelangesCrapel/file-424-1.pdf [03/06/2021].

CONSEIL DE L'EUROPE (2001). Cadre Européen Commun de Référence pour les Langues: apprendre, enseigner, évaluer. Strasbourg: Division des Politiques Linguistiques. https://rm.coe.int/16802fc3a8 [03/06/2021].

CONSEIL DE L'EUROPE (2009). Relier les examens de langues au Cadre européen commun de référence pour les langues: Apprendre, enseigner, évalver (CECRL). Un manuel. Strasbourg : Division des Politiques Linguistiques.

https://rm.coe.int/1680667a2e [03/06/2021].

CONSEIL DE L'EUROPE (2018). Cadre Européen Commun de Référence pour les Langues: apprendre, enseigner, évaluer. Volume complémentaire avec de nouveaux descripteurs. Strasbourg: Programme des Politiques linguistiques. https://rm.coe.int/cecr-volume-complementaire-avec-de-nouveauxdescripteurs/16807875d5 [03/06/2021].

Eemeren, F. H. \& GroOtendorst, R. (2004). A Code of Conduct for Reasonable Discussants Chapitre 8. A systematic Theory of Argumentation: The PragmaDialectical Approach. Cambridge : Cambridge University Press, pp. 187-196.

ESCANDELL VIDAL, M. V. (1996). Introducción a la pragmática. Barcelone : Ariel Lingüística.

GRIzE, J.-B. (1990). Logique et langage. Gap : Ophrys.

HIDDEN, M.-O. (2009a, du 25 au 29 mars). Variabilité culturelle des genres et enseignement de la production écrite en langue étrangère [communication]. Colloque International Les langues-cultures à l'Université, Bucarest. https://hal.archives-ouvertes.fr/hal-01850325 [03/06/2021].

HIDDEN, M.-O. (2009b). Pour une approche interculturelle des genres discursifs. Synergies Pays germanophones, 2, pp. 101-111.

http://gerflint.fr/Base/Paysgermanophones2/paysgermanophones2.html [03/06/2021].

MARcos García, M. J. (2009). El proceso de evaluación por competencias en las lenguas extranjeras. In I. Rodríguez Escanciano, Estrategias de innovación en el nuevo proceso de evaluación del aprendizaje. Valladolid: Universidad Europea Miguel de Cervantes, pp. 357-368.

TAGliante, C. (2009). L'évaluation et le Cadre Européen Commun (nouvelle éd.). Paris : CLE International.

\section{Sitographie}

http://formation-delfdalf.ciep.fr/ [03/06/2021].

https://examenes.cervantes.es/es/dele/examenes/bl [03/06/2021].

https://www.france-education-international.fr/exemples-sujets-b1-tp [03/06/2021]. 


\section{Annexes}

\section{Épreuves écrites}

DELF B1 - Sujet de démonstration Tout Public

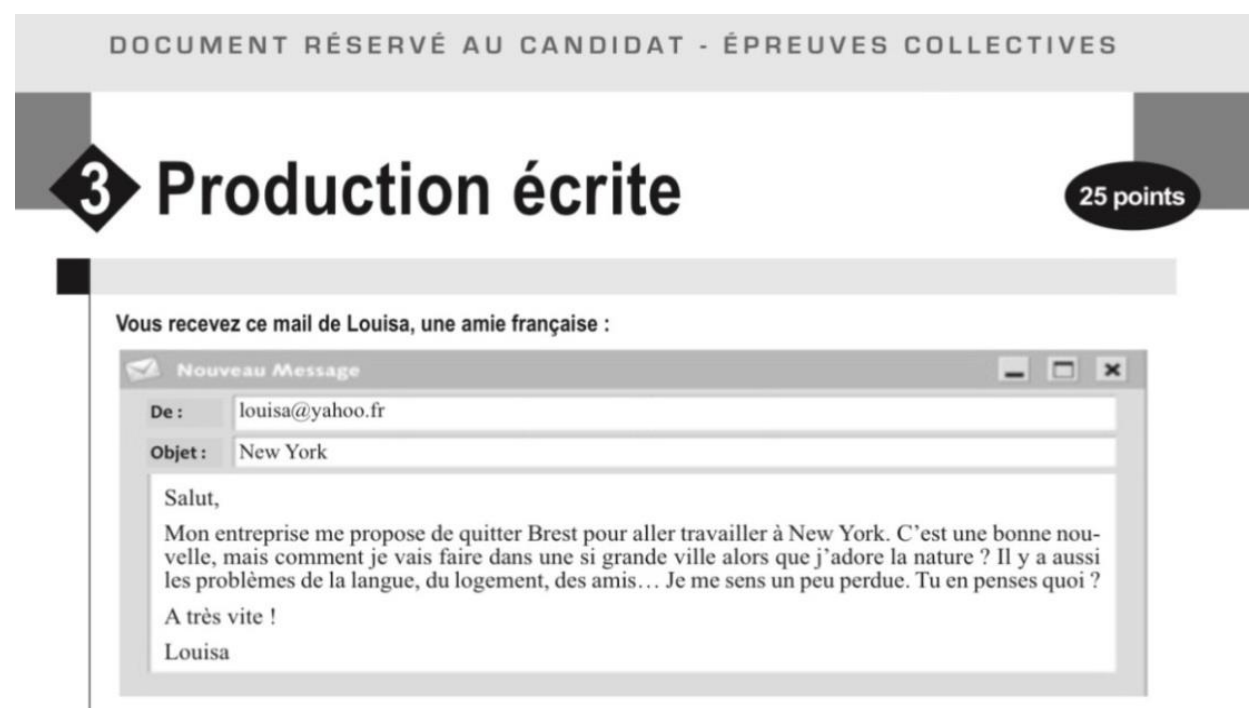

Vous répondez à Louisa. Vous lui donnez votre opinion en lui donnant des exemples d'expériences diverses. (160 mots minimum)

Disponible sur https://www.france-education-international.fr/exemples-sujets-b 1-tp

\section{DELE B1}

Expresión e interacción escritas
Página 3

\section{OPCIÓN 2}

Lea el siguiente mensaje que aparece en la página web del Ayuntamiento de su ciudad: FIESTAS DE LA CIUDAD

Invitamos a todos los ciudadanos que lo deseen a participar en el foro de la página web del Ayuntamiento. Pueden participar opinando sobre las fiestas del año pasado y comentando qué actividades les gustaría que se programaran en las próximas fiestas de la ciudad.

Redacte un texto para enviar al foro en el que deberá:

- presentarse;

- decir a qué actividades asistió el año pasado durante las fiestas;

- explicar qué actividades de las fiestas del año pasado le gustaron más y cuáles menos y por qué;

- $\quad$ proponer varias actividades para las próximas fiestas.

Disponible sur https://examenes.cervantes.es/es/dele/examenes/bl 


\section{Épreuves orales}

DELF B1 - Sujets de démonstration Tout Public : exercice 3

\section{MONOLOGUE SUIVI - 5 à 7 minutes}

Préparation : 10 minutes

Objectifs :

- Identifier un sujet de discussion à partir d'un texte déclencheur.

- Donner son opinion et en débattre avec l'examinateur.

Consigne au candidat :

Vous dégagerez le thème soulevé par le document ci-dessous. Vous présenterez ensuite votre opinion sous la forme d'un court exposé de 3 minutes environ. L'examinateur pourra vous poser quelques questions. Vous tirez au sort deux sujets et en choisissez un.

AU CHOIX DU CANDIDAT APRÈS TIRAGE AU SORT DE DEUX DOCUMENTS :

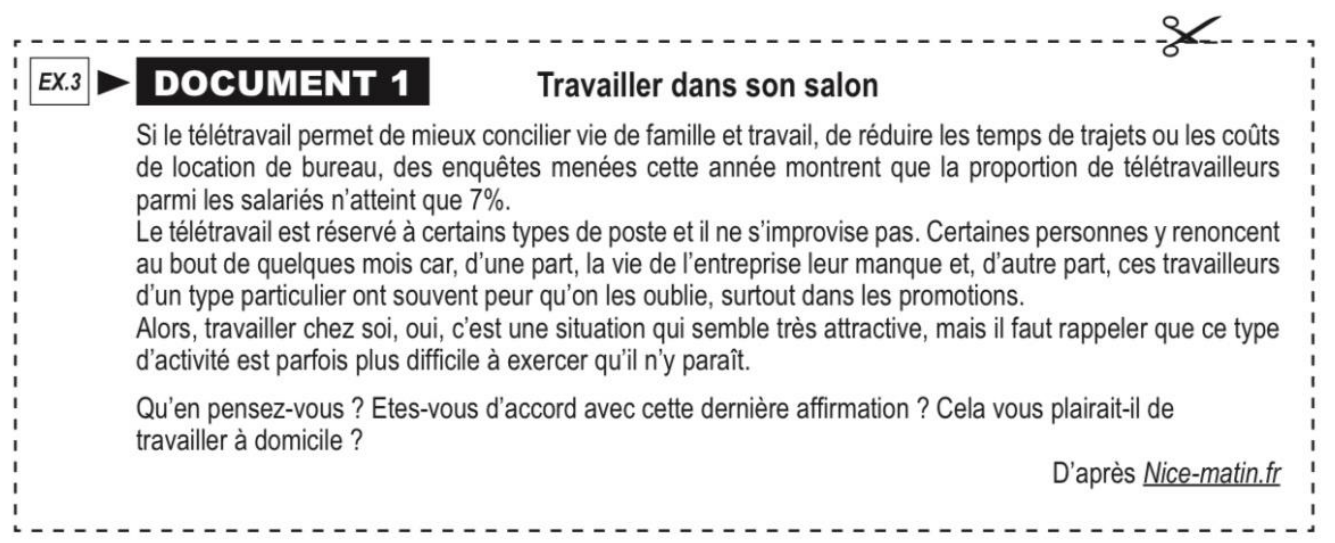

Disponible sur https://www.france-education-international.fr/exemples-sujets-b 1-tp 


\section{DELE B1}

\begin{tabular}{|c|c|}
\hline$e$ & $\begin{array}{c}\text { Expresión e interacción orales } \\
\text { Página 2 }\end{array}$ \\
\hline
\end{tabular}

Tarea 1

\section{Instrucciones}

Le proponemos dos temas con algunas indicaciones para preparar una exposición oral. Elija uno de ellos.

Tendrá que hablar durante $\mathbf{2}$ o $\mathbf{3}$ minutos sobre el tema elegido. El entrevistador no intervendrá en esta parte de la prueba.

\section{EJEMPLO DE TEMA:}

Hable de un país donde le gustaría vivir o del país ideal para vivir, en su opinión.

Incluya información sobre:

- $\quad$ qué país es; por qué le gustaría vivir allí o por qué cree que ese sería el país ideal para vivir;

- desde cuándo le gusta ese país; qué es lo que más le gusta y qué es lo que menos le gusta de ese país;

- qué le gustaría hacer allí; cuándo y con quién le gustaría vivir allí;

- $\quad$ experiencias de otras personas que hayan vivido en ese país.

No olvide:

- diferenciar las partes de su exposición: introducción, desarrollo y conclusión final;

- $\quad$ ordenar y relacionar bien las ideas:

- justificar sus opiniones y sentimientos.

\section{Tarea 2}

\section{Instrucciones}

Cuando haya terminado su exposición (Tarea 1), usted deberá mantener una conversación con el entrevistador sobre el mismo tema durante 304 minutos.

\section{EJEMPLOS DE PREGUNTAS DEL ENTREVISTADOR:}

- ¿Ha vivido usted en diferentes países/ciudades? ¿Dónde?

- De los sitios en que ha vivido, ¿cuál es el que más le ha gustado? ¿Por qué?

- ¿Cuáles son los aspectos más importantes en su opinión para decidir vivir en un determinado país / ciudad? ¿Por qué?

Disponible sur https://examenes.cervantes.es/es/dele/examenes/bl 